\section{Mirror-image stimulation}

\section{and tonic immobility in chickens*}

GORDON G. GALLUP, JR.

Tulane University, New Orleans, La. 70118

Group-reared chicks manually restrained in the presence of an unrestrained conspecific remained immobile for less time than birds restrained in isolation. On the other hand, chicks restrained in front of their own reflection in a mirror showed exaggerated tonic immobility reactions. Using differently prepared dead conspecifics as stimuli, it was determined that the reason a mirror prolongs the immobility episode relates to the reflected gaze of the "other" immobilized bird.

Animal hypnosis is represented by an almost catatonic-like state of physical or tonic immobility that usually occurs in response to some form of manual restraint. Contrary to what the name implies, the reaction seems to represent an innate fear response that, under natural conditions, serves as a predator defense (Gallup, Nash, Donegan, \& McClure, 1971; Gallup, Nash, Potter, \& Donegan, 1970; Ratner, 1967).

Using group-reared chicks, Salzen (1963) found that the presence of other unrestrained chickens in the testing situation served to reduce the duration of tonic immobility compared to birds restrained in isolation. On the basis of these findings, Salzen states that "there is no doubt that the primary cause of the immobility reaction is the sudden isolation of the chick from its social environment $[1963$, p. 70$]$.' Moreover, since in most immobility studies chicks are typically tested in isolation, Salzen feels that the reaction in domestic fowl can be attributed to fear associated with separation from imprinted stimuli.

Although Salzen's data fit in well with others that seem to implicate immobility as a fear-mediated reaction (e.g., Gallup, Rosen, \& Brown, 1972), the question arises as to how a chicken might respond to the presence of an immobilized, rather than a free moving, companion in the testing situation. The present study was designed to test this additional effect of social stimulation on animal hypnosis.

\section{EXPERIMENT 1}

The first experiment was designed to simulate confrontation with an immobilized conspecific by placing a mirror in front of an immobilized bird. The other obvious possibility might be to restrain two birds simultaneously; however, the inherent problem with this procedure relates to the fact that termination of immobility by one bird might precipitate termination by the

*Appreciation is expressed to $\mathrm{E}$. $\mathrm{T}$. Swanson and M. K. McClure for help in data collection. other due to the abrupt change in visual stimulation. Doty (1969) has convincingly shown that changing visual stimulation tends to reduce the duration of tonic immobility.

\section{Method}

The Ss were 36 Production Red chickens (Gallus gallus) obtained from a local hatchery at 2 days of age. During rearing, all birds were kept in commercial brooders which provided free access to Purina Chick Chow (Growena) and water. The photoperiod consisted of $14 \mathrm{~h}$ of artificial light per day, with room temperature held constant at approximately $22^{\circ} \mathrm{C}$.

The apparatus consisted of a rectangular plywood box measuring $91 \times 32 \times 46 \mathrm{~cm}$. Approximately $30 \mathrm{~cm}$ from one end, the box was fitted to accommodate a $31 \times 40 \mathrm{~cm}$ glass mirror or a comparably dimensioned piece of clear Plexiglas, which thereby provided for two separate compartments. The large chamber was used for testing and the other for purposes of housing a stimulus animal. Lighting was provided by overhead fluorescent lights, and the level of illumination in each chamber

At approximately 3 weeks of age, all chicks were divided randomly into three groups for testing. Birds from one group were individually placed into the testing side of the box and given 15 sec of manual restraint using the procedure described by Gallup, Nash, \& Wagner (1971). With the Plexiglas in position and the adjoining chamber empty, each bird was positioned $15 \mathrm{~cm}$ in front of the Plexiglas during manual restraint. For birds that did not become immobile following initial exposure to manual restraint, successive 15 -sec inductions until a maximum of five inductions had been administered.

In the second group, individual birds were immobilized in the visual presence of an unrestrained conspecific of the same age, separated from the $\mathbf{S}$ by the Plexiglas partition. For chickens in the third group, the was approximately the same. were given until immobility ensued or
Plexiglas was removed and all Ss were individually immobilized in front of their own reflection.

The birds in all three groups were laterally restrained on their right sides facing either the Plexiglas or the mirror. Duration of immobility was timed with a stopwatch from the time manual restraint was terminated until the bird rose to its feet. During each immobility episode, the E sat quietly on a chair about $1 \mathrm{~m}$ away and avoided making direct eye contact with the S.

\section{Results}

The effect of the different stimulus conditions on mean duration of immobility is portrayed in Fig. 1. As can be seen, the presence of a live conspecific tended to attenuate the reaction relative to birds restrained in isolation, whereas the mirror served to accentuate it. A simple between-groups analysis of variance performed on the data yielded a significant effect $(F=6.15, \mathrm{df}=2 / 33$, $\mathrm{p}<.01$ ). Thus, Salzen's finding of reduced immobility in the presence of conspecifics was replicated, but in light of the mirror data, it is not the presence of a conspecific per se that appears to account for the reduction.

\section{EXPERIMENT 2}

Since, in a variety of situations, duration of immobility seems to be related to procedures designed to manipulate fear, the major question raised by the first experiment is why a mirror should scare chickens. In other, more conventional situations, mirrors have been shown to have what might appear to be incentive properties for young chicks (Gallup, Montevecchi, \& Swanson, 1972).

The second experiment was designed to determine why visual access to a chick's own reflection during the immobility episode should exaggerate the response by a factor of over three times relative to Ss tested in social isolation. One possible way to

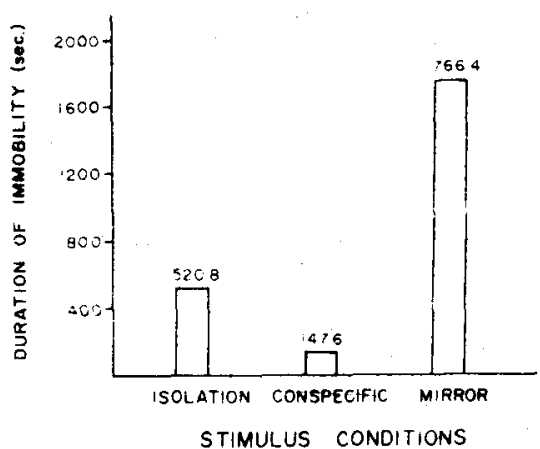

Fig. 1. Mean duration of tonic immobility in preadolescent chickens as a function of the social stimulus conditions in effect during testing. 
interpret the mirror data might be to hypothesize that the reflection of "another" immobilized bird serves to signal potential danger or predation. To test this notion, a freshly killed conspecific was placed into the test situation and used as a stimulus animal.

\section{Method}

The Ss consisted of 24 Production Red chicks obtained and maintained similarly to those in the previous study.

At 3 weeks of age, the chicks were divided randomly into two groups. The birds in one group were run under conditions that were identical to those in effect for the control group in the first experiment. In the second group chicks were individually immobilized in the presence of a comparably aged conspecific, which was sacrificed by means of ether inhalation and was positioned on its left side, $15 \mathrm{~cm}$ on the other side of the Plexiglas partition, in a posture resembling that of an immobilized bird.

\section{Results}

The visual presence of a dead conspecific produced no detectable effect on the immobility reaction. The mean duration of immobility for birds in the control group was $643.1 \mathrm{sec}$, whereas for chicks given visual access to the dead conspecific, the average was $666.6 \mathrm{sec}(F<1.0)$. Thus, the visual presence of a dead companion contributed virtually nothing to the duration of the immobility episode. EXPERIMENT 3

One possible difference between the reflection of an immobilized bird and a dead companion positioned in a posture approximating an immobilized conspecific might be whether or not the stimulus animal has its eyes open. Looking at one's reflection in a mirror presupposes that the image will be one of an animal with its eyes open, while the eyelids of dead chickens are typically closed.

Considerable data with chickens already exist which show that as stimuli in interspecific encounters, eyes exert a profound effect on immobility reactions. For example, Gallup, Nash, Donegan, \& McClure (1971) found that covering the eyes of a stuffed hawk with small pieces of black tape dramatically reduced its overall effectiveness as a stimulus for sustaining immobility. Similarly, Gallup, Cummings, \& Nash (1972) have shown that having the $\mathrm{E}$ stare directly at a restrained chick is an effective method for prolonging the immobility reaction. Artificial eyes suspended over immobilized birds (Gallup, Nash, \& Ellison, 1971) also greatly enhance the immobility episode in chickens.

In an attempt to approximate the exaggerated effect of a mirror, chicks in the third experiment were immobilized in the presence of a dead companion whose eyelids had been sutured open to expose the eyes.

\section{Method}

The Ss were 24 Production Red chicks obtained and maintained as in the previous experiments.

After attaining 3 weeks of age, the Ss were divided randomly into two groups for testing. Using the same testing chamber, chicks in one group were tested in isolation as controls. The remaining 12 birds were immobilized in the visual presence of a dead conspecific, whose eyelids had been carefully sutured open, that was positioned $15 \mathrm{~cm}$ behind the Plexiglas in a typical immobilized posture.

\section{Results}

The presence of a dead bird with its eyes open increased the duration of tonic immobility appreciably, with chicks showing a mean of 1,316.3 sec of self-paced immobility as compared to $591.7 \mathrm{sec}$ for control birds. A simple between-groups analysis of variance showed this difference to be statistically significant $(F=4.36$, $\mathrm{df}=1 / 22, \mathrm{p}<.05)$.

\section{DISCUSSION}

Data from the first experiment replicate those obtained by Salzen (1963) in showing that the presence of a free-moving conspecific in the testing situation produced a sharp decline in the duration of immobility shown by group-reared birds. However, an interpretation of tonic immobility as being primarily due to sudden social isolation was not clearly supported by the mirror data. Birds immobilized in the presence of their own reflection remained immobile almost 12 times longer than chicks tested in the presence of a companion (see Fig. 1) and over 3.5 times longer than chicks tested in isolation. Thus, certain types of atypical social stimulation may be more effective for prolonging immobility than simple separation from an imprinted stimulus.

The remaining two experiments were designed to determine why the reflection of an immobilized chick produced such an exaggerated hypnotic reaction for birds in the first experiment. In the second study, it' was found that the visual presence of a dead companion had virtually no effect on immobility. The data from Experiment 2 further document the notion that the simple presence or absence of other birds does not necessarily affect immobility.

In a final attempt to identify the critical factor responsible for prolonging the reaction in the presence of a mirror, it was reasoned that the animal's own reflected eyes might be important. To test this notion, the third experiment employed a dead conspecific that had its eyelids sutured open as the stimulus animal. In concert with other findings (e.g., Gallup, Nash, \& Ellison, 1971), the effect of making the dead bird's eyes visible was to appreciably enhance the duration of immobility, as compared to control data and the data obtained with dead stimulus birds in Experiment 2. This is not to imply that the eyes of another chicken are inherently fear-producing, but rather that the effect of eye contact in an intraspecific encounter is probably contextually determined.

As a check on the effect of a mirror vs that of a dead conspecific with its eyes open, two additional groups of 12 Ss each were immobilized under one of these conditions using comparable procedures in a fourth experiment. The obtained results replicate the original findings, with a mean of $1,736.9 \mathrm{sec}$ for the mirror group and a mean of $1,250.2$ sec for chicks in the other group. Although not significant $(F=1.30, \quad \mathrm{df}=1 / 22)$, the difference between these two conditions may be due to the fact that the pupil in the eyes of dead chickens was observed to dilate appreciably shortly after death, and therefore the eyes appear slightly atypical.

\section{REFERENCES}

DOTY, R. L. The effect of environmental movement upon the duration of tonic immobility in bobwhite quail. Psychonomic Science, 1969, 16, 48-49. GALLUP, G. G., JR., CUMMINGS, W. H., \& NASH, R. F. The experimenter as an independent variable in studies of animal hypnosis in chickens (Gallus gallus). Animal Behaviour, 1972, 20, 166-169.

GALLUP, G. G., JR., MONTEVECCHI, W A.. SWANSON, E. T. Motivational properties of mirror-image stimulation in the domestic chicken. Psychological Record, 1972, in press.

GALLUP, G. G., JR., NASH, R. F. DONEGAN, N. H., \& MCCLURE, M. K. The immobility response: A predator-induced reaction in chickens. Psychological Record, 1971, 21, 513-519. GALLUP, G. G., JR., NASH, R. F., \& ELLISON, A. L., JR. Tonic immobility as a reaction to predation: Artificial eyes as a fear stimulus for chickens. Psy chonomic Science, $1971,23,79-80$.

GALLUP, G. G., JR., NASH, R. F., POTTER, $R$ J \& DONEGAN N $H$ Effect of varying conditions of fear on immobility reactions in domestic chickens (Gallus gallus). Journal of Comparative \& Physiological Psychology $1970,73,442-445$.

GALLUP, G. G., JR., NASH, R. F., \& WAGNER, A. M. The tonic immobility reaction in chickens: Response characteristics and methodology. Behavior Research Methods \& Instrumentation, 1971, 3, 237-239.

GALLUP, G. G., JR., ROSEN, T. S., \& BROWN, C. W. Effect of conditioned fear 
on tonic immobility in domestic chickens. Journal of Comparative \& Physiological Psychology, 1972, 78, 22-25.
RATNER, S. C. Comparative aspects of hypnosis. In J. E. Gordon (Ed.), Handbook of clinical and experimental hypnosis. New York: Macmillan, 1967.
Pp. 550-587.

SALZEN, E. A. Imprinting and the immobility reactions of domestic fowl Animal Behaviour, 1963, 11, 66-77.

\section{NOTES \& NEWS}

Ricardo Dobson, who, for the past three years, was Assistant Professor at Mary Washington College of the University of Virginia, is now Associate Senior Research Psychologist at General Motors Research Laboratories in Warren, Michigan.

R. B. Freeman, Jr., formerly Professor at The Pennsylvania State University, is now Professor of Psychology at the University of Konstanz, Germany. He will be teaching in the fields of experimental and physiological psychology and continuing research on the psychophysics and electrophysiology of vision and visual perception.

Drs. Jacquelin and Robert Goodnow are now affiliated with the School of Education, Macquarie University, North Ryde, Australia. Jacquelin Goodnow, formerly Associate Research Professor at George Washington University, is now a professor of psychology and is doing research in cross-cultural studies. Robert Goodnow, formerly President of the Psychological Assessment Association, has switched from industrial psychology to environmental psychology/landscape architecture.

John D. Gould, after a sabbatical year as a visiting associate professor at Carnegie-Mellon University, has now returned to the Thomas J. Watson Research Center of $I B M$ where he is Manager of the Psychology Group.

Marshall Haith has accepted a position at the University of Denver as Professor and Head of the Behavior Development Laboratory in the University.
Department of Psychology. Formerly he was a lecturer in the Department of Social Relations, Harvard University.

Willem J. M. Levelt, who spent the last-academic year at the Institute for Advanced Study, Princeton, is now back at his permanent position as Professor of Psychology, Nijmegen University, The Netherlands.

Gardner Lindzey, who has been at The University of Texas in Austin for the past few years, and most recently at the Center for Advanced Studies in the Behavioral Sciences at Stanford, will become Chairman of the newly reorganized Department of Psychology and Social Relations at Harvard

R. D. Luce, recently from the Institute for Advanced Study at Princeton, is now Professor of Social Sciences at the University of California, Irvine.

Dr. Joan C. Martin has accepted the position of Associate Professor, Department of Psychiatry and Behavioral Sciences, University of Washington, Seattle. Dr. Martin was formerly Assistant Professor of Medical Psychology at Duke University, 1969-1971. Most recently she was a grants associate fellow, National Institutes of Health, 1971-1972.

George McConkie has been on sabbatical leave for the past year doing research at the Artificial Intelligence Laboratory at The Massachusetts Institute of Technology. He will return to his regular position as Associate Professor in the Department of
Education at Cornell University on September 1.

Richard F. Nash who has just received his $\mathrm{PhD}$ in psychology from Tulane University, has accepted a position as Assistant Professor in the Psychology Department at Marquette University. His major area of interest is animal behavior.

Larry M. Raskin is returning to Purdue University as Associate Professor in the Department of Child Development and Family Life after a year's leave of absence at the Division for Disorders of Development and Learning, University of North Carolina.

Edward J. Rowe, formerly a postdoctoral fellow in the Department of Psychology, University of Alberta, was appointed Assistant Professor of Psychology at Memorial University in St John's, Newfoundland.

Michel Treisman has resigned from the position of Head of the Department of Psychology at the University of Reading to accept a position in the Department of Experimental Psychology, Oxford University.

Anthony A. Wright, who has been at The University of Texas at Austin, is now Assistant Professor of Neural Sciences at The University of Texas Graduate School of Biomedical Sciences, Texas Medical Center, Houston. The function of the Department of Neural Sciences is to educate a small number of graduate students and to provide an environment for interdisciplinary and collaborative research. 